\section{P-63 OACC ONE YEAR ON}

Michaela Sen, Claude Chidiac. Saint Francis Hospice, Romford, UK

\subsection{6/bmispcare-2018-hospiceabs.88}

Background The Outcome Assessment Complexity Collaborative (OACC) is a validated suite of outcome measures that is aimed to measure, demonstrate and improve patients' and caregivers' outcomes. Following the initial phase of its implementation at Saint Francis Hospice utilising Kotter (2007) eight step model of change, the established Steering Group continues to sustain and institute change, with a focus on providing effective leadership to oversee the embedding and anchoring of the measures within all clinical services.

Aim To embed and anchor the OACC Suite of Outcome Measures within all clinical services at Saint Francis Hospice.

Method The project lead from each service provides support and any problems identified are reported to and addressed by the Steering Group. Compliance has been monitored through audit. Relevant policies, standards of practice and assessment documentation have been reviewed and revised. Training is incorporated within the clinical induction programme for new staff. Constant feedback from clinical staff and updates on IT reporting developments continue to be sought, recorded and brought to the Steering Group.

Results Some challenges have been identified relating to IT reporting, one measure has required review to ensure it fits and supports the demand of the workload within a team and compliance with the timing of the use of the measures is also under review for another individual team. All clinical teams are incorporating the use of OACC within their practice and this is leading to a transformational change in both the delivery of service for individual teams and for the organisation as a whole.

Conclusion By displaying effective leadership, supporting review and development of processes and reinforcing change, OACC is being successfully used at Saint Francis Hospice and is playing a crucial role in further improving the quality, efficiency and availability of palliative care for our service users.

\section{\begin{tabular}{|l|l}
\hline P-64 WITH US NOT TO US \\
\hline
\end{tabular}}

Will McLean, Jill Cook. Children's Hospices Across Scotland, Edinburgh, UK

\subsection{6/bmjspcare-2018-hospiceabs.89}

In response to CHiSP (Fraser, Jarvis, Moran et al., 2015) an integral part of CHAS' strategy is to offer practical help and sibling support to families caring for a child with a life-shortening condition in their own homes. We embedded an outcome measurement approach to the pilot from planning through management to reporting as understanding the impact of the support offered was a crucial underpinning of the project.

In developing our impact measurement approach we identified key stakeholders. We listened to what families and volunteers were telling us at pre-planning stage and developed outcomes. We designed a monitoring system using indicators from the Getting It Right For Every Child (GIRFEC) wellbeing framework. This framework, designed by the Scottish Government - a major funder of CHAS - is widely recognised allowing us to easily disseminate findings and communicate with a significant investor. Indicators informed outcome 'webs', bespoke data collection tools applied through semi-structured interviews with stakeholders throughout the pilot. A variety of activities, including Lego and board games, were used to support the interview process with siblings supported by the pilot, while completing an easy to understand child's web. Interviews were used as a volunteer management tool, helping with supervision, identifying challenges and developing the pilot.

The pilot has been recommissioned as we have proved demonstrable value to the families and volunteers, whose involvement in the planning and decision making process developed a service that really works. A service provided 'with us, not to us' was important to families who had a real say in their care. We were able to effectively communicate our success to wider audience in terms that were recognisable and familiar and are now in discussions with a potential funder to scale up this project from the pilot phase, initially in four local authority areas, to cover the whole of Scotland.

\section{P-65 HOSPICE AND HOSPITAL PALLIATIVE OUTCOME MEASURES PROJECT}

${ }^{1}$ Claire State, ${ }^{2}$ Emma Newbold, ${ }^{1}$ Fiona Chiplen, ${ }^{1}$ Amanda Gough. ${ }^{1}$ Weston Hospicecare, Weston-super-Mare, UK; ${ }^{2}$ Weston General Hospital, Weston-super-Mare, UK

\subsection{6/bmispcare-2018-hospiceabs.90}

It is established that using Palliative Outcome Measures (POMs) leads to an improvement in health related quality of life. The hospital and hospice palliative care teams identified that different assessment tools were being used across both settings and recognised this was a barrier to communication. Working together using the same assessment tools would improve communication, ensure consistency and promote patient centred care.

Our aim was to implement POMs (Phase of Illness, Australia-modified Karnofsky Performance Status (AKPS) and Integrated Palliative Outcome Scale (IPOS) across both settings in order to provide a consistent approach.

Using the POMs we wanted to capture changes in health status, promote improved communication between professionals, aid decision making, ensure patient driven assessment and planning and monitor if IPOS scores show an improvement in patient symptoms, wellbeing and quality of life.

Joint meetings and training have already taken place with the plan to use quality improvement methodology to show whether:

- POMs are being used between settings and effectively improve communication.

- The level of patient compliance and whether it is user friendly

- Early recognition of patient symptoms and wellbeing leads to prompt interventions

- If patients admitted to hospital, hospice named nurse informs palliative care team of Phase of Illness and AKPS score.

- On hospital discharge letter to hospice/GPs/District Nurses the Phase of Illness and AKPS score is recorded.

- An IPOS form is given to all known and new hospice patients on discharge from WGH.

- Hospice to use PCOMs at MDT, and IPU meeting and record on handover sheets and electronic patient system.

- Using the QI methodology the first three PDSA cycles were formulated, Cycle 1\& 2 being joint meetings and training and a Go Live date was set. Cycle 3 plans to undertake the first review and audit after three months, when further cycles will be planned. 